\title{
Hydrodynamic Analysis of Different Finger Positions in Swimming: A Computational Fluid Dynamics Approach
}

\author{
J. Paulo Vilas-Boas, ${ }^{1}$ Rui J. Ramos, ${ }^{2}$ Ricardo J. Fernandes, ${ }^{1}$ António J. Silva, ${ }^{3}$ \\ Abel I. Rouboa, ${ }^{3}$ Leandro Machado, ${ }^{1}$ Tiago M. Barbosa, ${ }^{4}$ and Daniel A. Marinho ${ }^{2}$ \\ 1 University of Porto/LABIOMEP; ${ }^{2}$ University of Beira Interior/CIDESD; \\ ${ }^{3}$ University of Trás-os-Montes and Alto Douro/CIDESD; ${ }^{4}$ Nanyang Technological University/CIDESD
}

\begin{abstract}
The aim of this research was to numerically clarify the effect of finger spreading and thumb abduction on the hydrodynamic force generated by the hand and forearm during swimming. A computational fluid dynamics (CFD) analysis of a realistic hand and forearm model obtained using a computer tomography scanner was conducted. A mean flow speed of $2 \mathrm{~m} \cdot \mathrm{s}^{-1} \mathrm{was}$ used to analyze the possible combinations of three finger positions (grouped, partially spread, totally spread), three thumb positions (adducted, partially abducted, totally abducted), three angles of attack $\left(\mathrm{a}=0^{\circ}, 45^{\circ}, 90^{\circ}\right)$, and four sweepback angles $\left(\mathrm{y}=0^{\circ}\right.$, $90^{\circ}, 180^{\circ}, 270^{\circ}$ ) to yield a total of 108 simulated situations. The values of the drag coefficient were observed to increase with the angle of attack for all sweepback angles and finger and thumb positions. For $y=0^{\circ}$ and $180^{\circ}$, the model with the thumb adducted and with the little finger spread presented higher drag coefficient values for $\mathrm{a}=45^{\circ}$ and $90^{\circ}$. Lift coefficient values were observed to be very low at $\mathrm{a}=0^{\circ}$ and $90^{\circ}$ for all of the sweepback angles and finger and thumb positions studied, although very similar values are obtained at $\mathrm{a}=45^{\circ}$. For $\mathrm{y}=0^{\circ}$ and $180^{\circ}$, the effect of finger and thumb positions appears to be much most distinct, indicating that having the thumb slightly abducted and the fingers grouped is a preferable position at $\mathrm{y}=180^{\circ}$, whereas at $\mathrm{y}=0^{\circ}$, having the thumb adducted and fingers slightly spread yielded higher lift values. Results show that finger and thumb positioning in swimming is a determinant of the propulsive force produced during swimming; indeed, this force is dependent on the direction of the flow over the hand and forearm, which changes across the arm's stroke.
\end{abstract}

Keywords: CFD, performance, sport, aquatics, propulsion.

The study of human swimming propulsion is one of the most complex areas of interest in sport biomechanics. ${ }^{1}$ Over the past decades, research in swimming biomechanics has evolved from the observation of subjects' kinematics to a basic flow dynamics approach, paralleling the line of research followed by scientists working on this subject in experimental biology., 2,3

Computational fluid dynamics (CFD) methodology can be considered an interesting numerical tool in the field of biomechanics as well as in engineering. This methodology has been used in the computational analysis of fluid flow in several research fields, including medicine, biology, industry, and sport. ${ }^{4-7}$ This numerical tool represents a branch of fluid mechanics that solves and analyzes problems involving fluid flow by means of computer-based simulations. Within the context of sports, studies suggest that numerical analysis could provide useful information regarding performance. In swimming, this methodology has been used to study the propulsive forces produced by the hands and forearms ${ }^{8-10}$ and the legs during swimming, ${ }^{11,12}$ as well as the magnitude of the drag forces resisting forward motion. ${ }^{13-15}$

J. Paulo Vilas-Boas, Ricardo J. Fernandes, and Leandro Machado are with the Faculty of Sport, CIFI2D, and Porto Biomechanics Laboratory (LABIOMEP), University of Porto, Porto, Portugal. Rui J. Ramos and Daniel A. Marinho are with the University of Beira Interior, CIDESD, Covilhã, Portugal. António J. Silva and Abel I. Rouboa are with the University of Trás-os-Montes and Alto Douro, CIDESD, Vila Real, Portugal. Tiago M. Barbosa is with the National Institute of Education, Nanyang Technological University, Singapore, and CIDESD. Address author correspondence to Daniel A. Marinho at dmarinho@ubi.pt.
With respect to propulsive forces, CFD can provide insight into basic issues concerning swimming technique that, based on the knowledge provided by experimental approaches, remain unclear or, at least, controversial. The relative position of the fingers and thumb during the underwater path of the stroke cycle is one of these issues. A large intersubject range of relative finger and thumb positions can be observed during training and competition. Concerning thumb position, some swimmers maintain the thumb adducted; whereas some show small thumb abduction, others show complete thumb abduction. Concerning finger spreading, some swimmers maintain the fingers grouped, others show a small separation between fingers, and still others maintain their fingers substantially separated. Moreover, the positions of the fingers and thumb appear to change throughout the stroke of elite swimmers, as if a "fine-tuning" system allows for the perception of the circumstantial advantages of different hand geometries.

The relevance of finger spreading and thumb abduction to propulsive force generation was previously highlighted by experimental and numerical approaches to human swimming propulsion (Table 1). It can be observed that only a few studies have been conducted to address this issue, and the results are far from being uncontroversial. Moreover, if the experimental findings obtained to date can be considered limited (considering sweepback angles and flow velocities), controversial, and inconclusive, the numerical approaches applied are even more limited. Marinho et $\mathrm{al}^{23}$ studied the effect of thumb position (adducted, 50\% abducted, and fully abducted) for a sweepback angle (y) of $0^{\circ}$ and for attack angles (a) of $0^{\circ}, 45^{\circ}$, and $90^{\circ}$, and Marinho et $\mathrm{al}^{24}$ complemented this work by analyzing the effect of finger position (grouped, 0.32-cm and 0.64-cm 


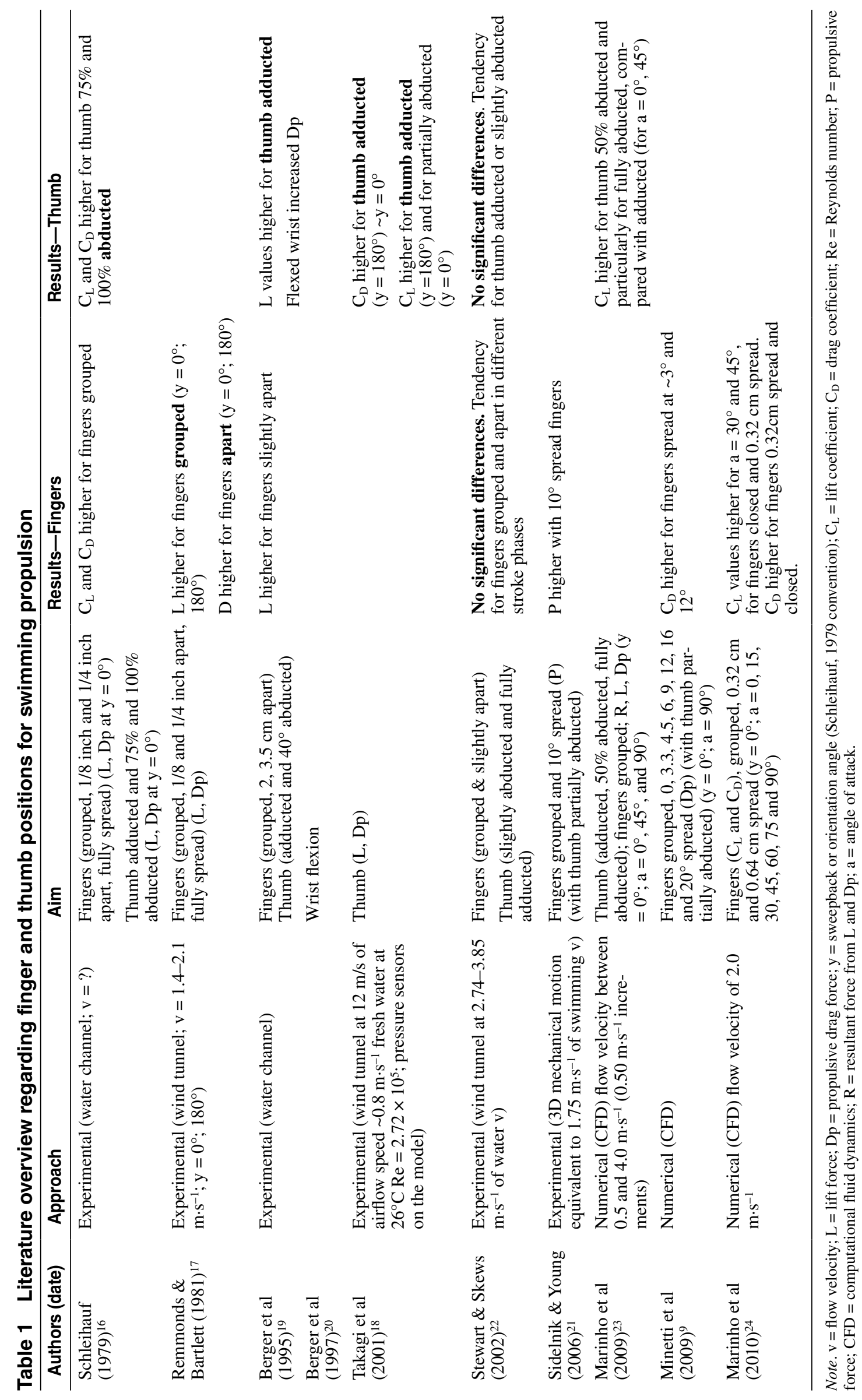


fingertip spreading) at $\mathrm{y}=0^{\circ}$ and attack angles varying from $0^{\circ}$ to $90^{\circ}$ in steps of $15^{\circ}$. Both studies indicated that the lift coefficient $\left(\mathrm{C}_{\mathrm{L}}\right)$ increases with thumb abduction and for positions in which the fingers are close together or slightly spread $(0.32 \mathrm{~cm})$ for $\mathrm{a}=30^{\circ}$ and $45^{\circ}$. The drag coefficient $\left(C_{D}\right)$ was also observed to be higher for positions in which the fingers were close together or $0.32 \mathrm{~cm}$ apart. These studies were conducted for isolated hand models and thus ignored the interaction of hand and forearm geometries. Minneti et $\mathrm{al}^{9}$ also numerically arrived at the same conclusion regarding finger spreading but with the thumb in a fixed position (partially abducted). Overall, the available knowledge base in this area of research is controversial and inconclusive. Moreover, numerical studies are scarce and limited in the number of sweepback angles and finger-thumb position combinations considered, and they ignore the geometric interactions between the hand and forearm.

Therefore, the purpose of this study was to analyze the effect on $\mathrm{C}_{\mathrm{L}}$ and $\mathrm{C}_{\mathrm{D}}$ of the hand and forearm at different sweepback and attack angles for different finger spreading and thumb abduction combinations.

\section{Methods}

\section{Three-dimensional Model}

Scanning. To obtain the geometry of the hand and forearm, cross-sectional scans of the hand and forearm of an elite swimmer were conducted using a Toshiba Aquilion 4 computer tomography scanner (Toshiba, Minato, Tokyo). The subject was an Olympiclevel swimmer who participated in the 2004 Olympic Games in Athens. The subject lay prone, with his right arm extended ahead and fully pronated. This procedure was repeated for different finger spread configurations (fingers grouped, fingers slightly spread [an intrafinger distance of $0.32 \mathrm{~cm}$, from tip to tip], and fingers greatly spread [0.64 cm, from tip to tip]) and different thumb positions (fully abducted [the angle between the forefinger and the thumb was $68^{\circ}$ ], partially abducted [the angle between the forefinger and the thumb was $30^{\circ}$ ], and adducted). ${ }^{14,24}$ The appropriate ethical committee of the institution where the study was performed approved this protocol, and the subject provided informed consent to participate.

Data Manipulation. The transformation of positions obtained from computer tomography scans into nodal coordinates in an appropriate coordinate system warrants the use of image-processing techniques. The image-processing program used in this study was Anatomics Pro (Anatomics, Kannapolis, Australia). This program allowed for the boundaries of the human segments to be obtained, creating a three-dimensional reconstruction of the hand and forearm. This step was also carried out using the software FreeForm (Sensable Technologies, Wobum, USA). Finally, the data were converted to an IGES format (*.igs) that could be read by
Fluent software (Ansys, Hanover, USA) to define the finite volume approach over the three-dimensional surfaces.

\section{Computational Fluid Dynamics}

Preprocessing. Nine models (Figure 1) were developed to analyze the different combinations of swimmer hand geometry: (i) thumb abducted and fingers greatly spread (Tapart-Fapart), (ii) thumb abducted and fingers slightly spread (Tapart-Fmiddle), (iii) thumb abducted and fingers close together (Tapart-Fgrouped), (iv) thumb partially abducted and fingers greatly spread (TmiddleFapart), (v) thumb partially abducted and fingers slightly spread (Tmiddle-Fmiddle), (vi) thumb partially abducted and fingers close together (Tmiddle-Fgrouped), (vii) thumb adducted and fingers greatly spread (Tgrouped-Fapart), (viii) thumb adducted and fingers slightly spread (Tgrouped-Fmiddle), and (ix) thumb adducted and fingers close together (Tgrouped-Fgrouped).

The whole domain was modeled using a hybrid mesh composed of prisms and tetrahedrons. Significant effort was made to ensure that the model would provide accurate results by decreasing the grid node separation in areas of high velocity and pressure gradient.

Solving Steady Flow. Steady-state computational fluid dynamics analyses were performed using the Fluent code, and $\mathrm{C}_{\mathrm{L}}$ and $\mathrm{C}_{\mathrm{D}}$ were computed for a flow velocity of $2.0 \mathrm{~m} \cdot \mathrm{s}^{-1}{ }^{23,25}$ The Fluent code solves flow problems by replacing the Navier-Stokes equations with discretized algebraic expressions that can be solved by iterative computerized calculations

Angles of attack (a) $0^{\circ}, 45^{\circ}$, and $90^{\circ}$ for the hand and forearm models with sweepback (y) angles ${ }^{16}$ of $0^{\circ}$ (thumb as the leading edge), $90^{\circ}$ (from fingertips to wrist), $180^{\circ}$ (ulnar flow), and $270^{\circ}$ (from wrist to fingertips) were used for the calculations (Figure 2). A total of 108 simulations were conducted, with the nine models tested for each combination of sweepback angle and angle of attack.

All numerical computational schemes were second-order, which provide a more accurate solution than first-order schemes. A turbulence intensity of $1.0 \%$ and a turbulence scale of $0.10 \mathrm{~m}$ were used. The water temperature was set to $28^{\circ} \mathrm{C}$, a density of 998.2 $\mathrm{kg} \cdot \mathrm{m}^{-3}$, and a viscosity of $0.001 \mathrm{~kg} \cdot(\mathrm{m} \cdot \mathrm{s})^{-1}$.

\section{Results}

The drag coefficients of the hand and forearm increased with the angle of attack for all sweepback angles and finger and thumb positions (Figure 3). Moreover, for sweepback angles of $\mathrm{y}=0^{\circ}$ and $180^{\circ}$, the model with the thumb grouped and the little finger spread (Tgrouped-Fmiddle) presented higher drag coefficient values for angles of attack of $\mathrm{a}=45^{\circ}$ and $90^{\circ}$ than those yielded by the other models. Sweepback angles of $\mathrm{y}=90^{\circ}$ and $270^{\circ}$ presented lower values of drag coefficient at angles of attack of $\mathrm{a}=0^{\circ}$ and $45^{\circ}$ than
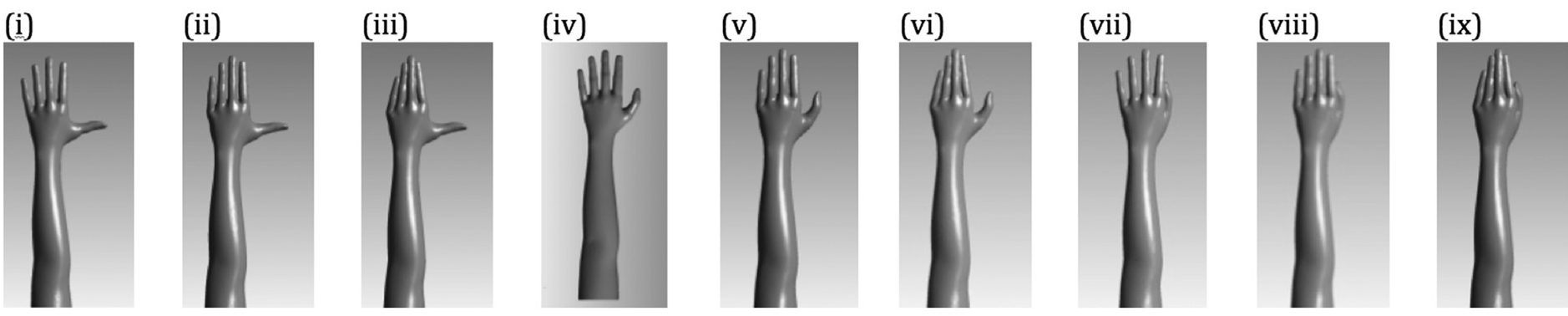

Figure 1 - Hand and forearm models used in this study. 

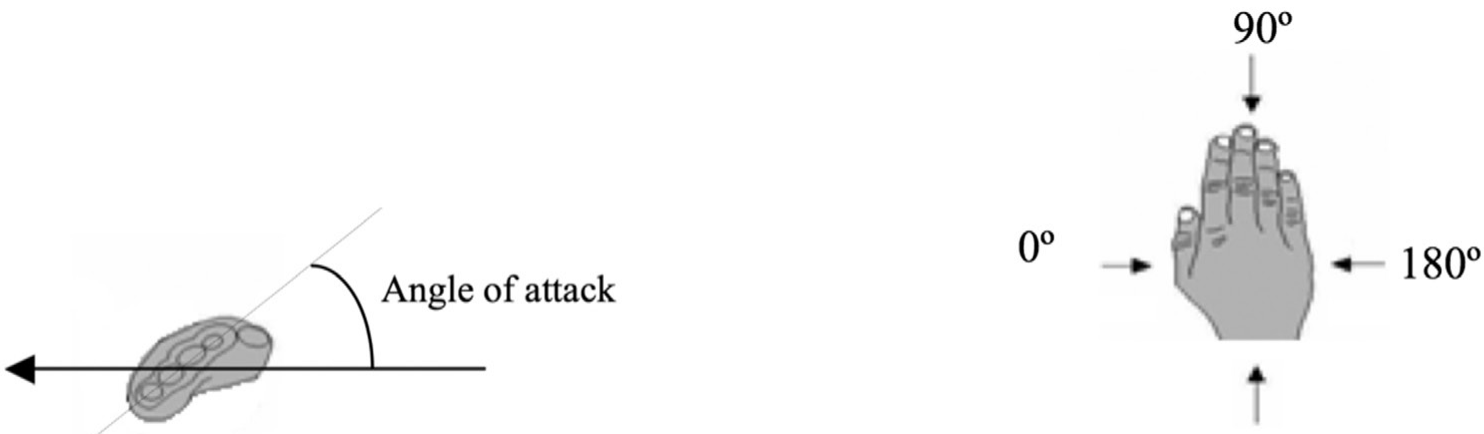

(i)

$270^{\circ}$

(ii)

Figure 2 - The angle of attack (i) and the sweepback angle (ii). The arrow represents the direction of the flow (adapted from Schleihauf ${ }^{16}$ ).
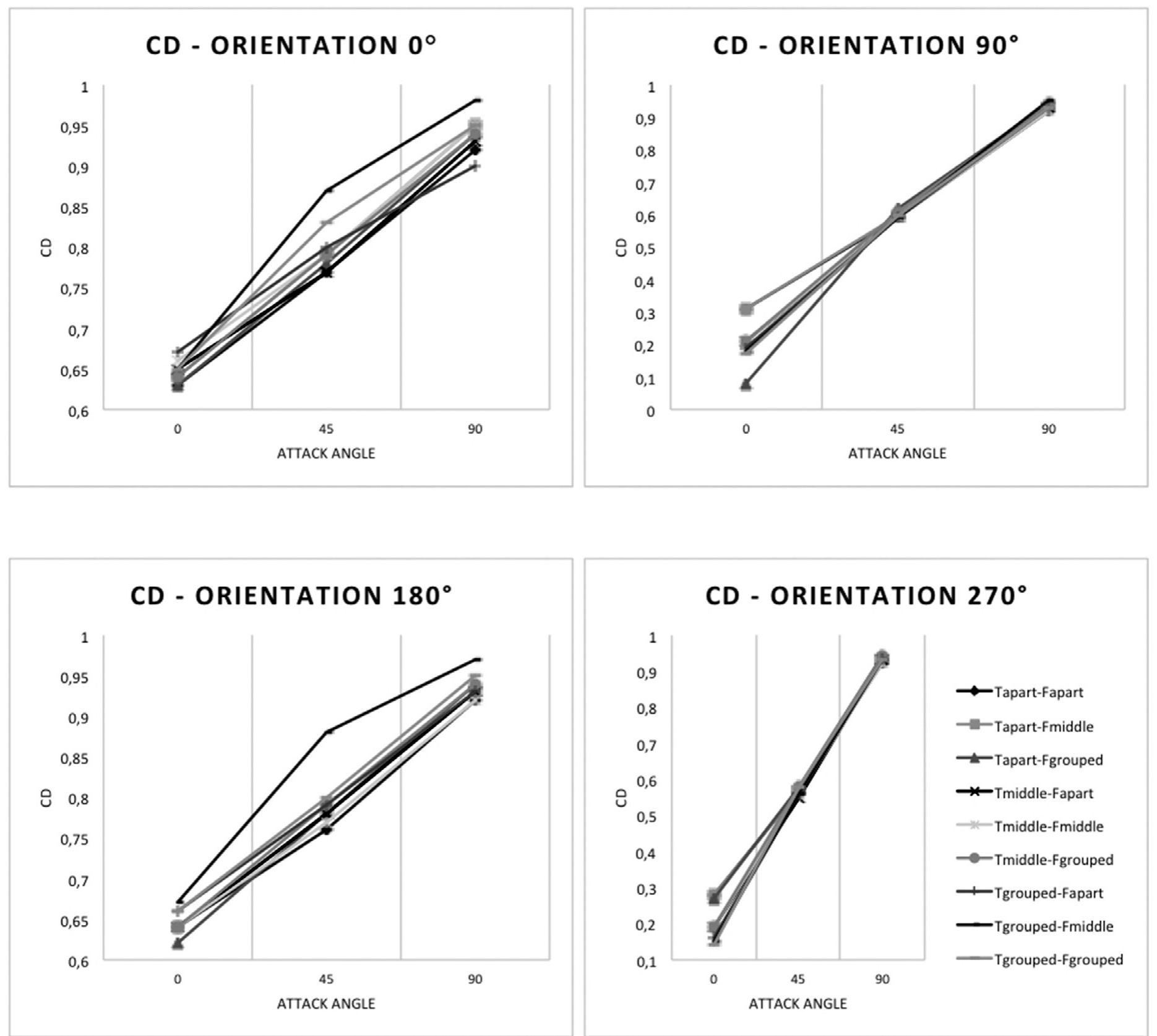

Figure 3 - Drag coefficient (CD) as a function of angle of attack (a) for different orientation or sweepback (y) angles. 
did the same hand and forearm models for sweepback angles of $y$ $=0^{\circ}$ and $180^{\circ}$.

The hand and forearm lift coefficients were very low at angles of attack of $\mathrm{a}=0^{\circ}$ and $90^{\circ}$ for all of the sweepback angles and finger and thumb positions studied (Figure 4). However, for sweepback angles of $\mathrm{y}=0^{\circ}$ and $180^{\circ}$, the lift neutrality was lower for $\mathrm{a}=0^{\circ}$ than for $\mathrm{a}=90^{\circ}$ but with relevant variations among different finger and thumb configuration models. On the other hand, for an angle of attack of $\mathrm{a}=45^{\circ}$, and also for all sweepback angles, the lift coefficient presented values much more similar to the hand and forearm drag coefficients. This finding was particularly evident for sweepback angles of $\mathrm{y}=90^{\circ}$ and $270^{\circ}$, for which the obtained lift coefficient values were much higher and more similar to the obtained drag coefficient values. In these hand and forearm flow orientations, only minor effects of finger spreading and thumb abduction were observed, with only slightly higher absolute values measured for the fingers grouped and thumb adducted. Conversely, for sweepback angles of $y=0^{\circ}$ and $180^{\circ}$, the effect of finger and thumb position was much more distinct, particularly at attack angles of $\mathrm{a}=45^{\circ}$, at which the resulting values were also higher and gained hydrodynamic relevance. At $y=180^{\circ}$, the thumb slightly abducted and the fingers grouped appeared to be the best position, followed by the thumb fully abducted and the fingers slightly spread and again the thumb fully abducted but with the fingers grouped. In contrast, at a sweepback angle of $\mathrm{y}=0^{\circ}$, a higher lift coefficient was also observed at an attack angle of $\mathrm{a}=45^{\circ}$ but with the thumb adducted and fingers slightly spread, followed by the thumb fully abducted and fingers grouped and the thumb partially abducted and fingers
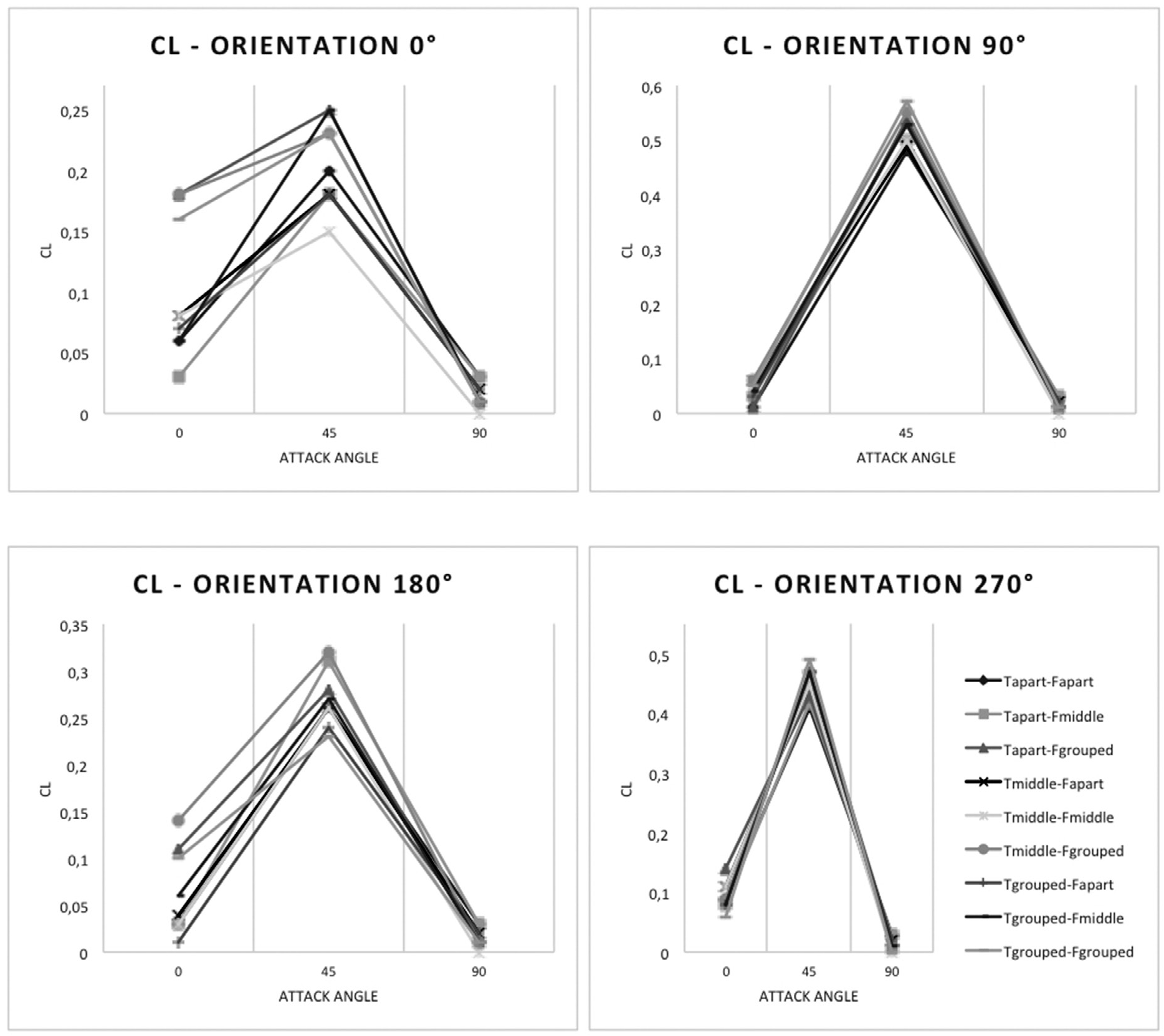

Figure 4 - Lift coefficient (CL) as a function of angle of attack (a) for different orientation or sweepback (y) angles. 
grouped. In this case, $C_{L}$ values similar to those obtained for the thumb adducted and fingers grouped were obtained, demonstrating that similar lift effects can be obtained with different thumb and finger positions for the same propulsive segment orientation using a single realistic human hand and forearm model.

\section{Discussion}

The aim of this study was to analyze the effect of different combinations of finger and thumb positions on the drag and lift coefficients of the hand and forearm at different sweepback and attack angles. The main results suggest that the extent to which the fingers are spread and the thumb position (more or less adducted or abducted) appear to play a secondary role in determining the drag and lift coefficient values of the hand and forearm together relative to the hand's attack and sweepback angles. At certain sweepback angles, the lift force may contribute similarly, or even more markedly, to swimming performance compared with the drag propulsive force but always at attack angles close to $\mathrm{a}=45^{\circ}$. This finding is based on the respective $C_{D}$ and $C_{L}$ values obtained, combined with the presumed cross-sectional area of the propulsive segment relative to the direction of the considered force, $S_{D, L}$, calculated using the Newtonian equations of the drag (D) and lift (L) forces:

$$
\begin{aligned}
& D=0.5 \rho C_{D} S_{D} v^{2} \\
& L=0.5 \rho C_{L} S_{L} v^{2}
\end{aligned}
$$

In the foregoing equations, $\rho$ represents water density and $v$ the flow velocity over the hand and forearm.

Based on the above-described results, the relative contribution of propulsive drag (Dp) and lift (L) to propulsion heavily depends on the arm stroke phase of each swimmer's technique, which determines the sweepback angle. This relationship also appears to hold for the effect of finger and thumb positions on the hydrodynamics of propulsion, which vary with $\mathrm{y}$. The relative effect of $\mathrm{L}$ and Dp on swimming propulsion is an old and very controversial issue. Following the seminal works of Counsilman, ${ }^{26}$ Schleihauf, ${ }^{16}$ and Wood, ${ }^{27}$ researchers such as Maglischo ${ }^{28}$ strongly advocated for the relevant role of lift. Afterward, however, concerns were raised $^{29}$ that, despite being mostly theoretical and intuitive, led authors such as Maglischo ${ }^{30}$ to radically change their perspective (fortunately Maglischo ended with a more balanced position in the last edition of his book ${ }^{31}$ ). Our results are not sufficient to resolve this issue, but they strongly support the possible influence of lift force on swimming propulsion. This effect might not be as strong as initially supposed but may be relevant and should not be ignored in technical terms when technique is described, taught, or trained. Indeed, the hand and forearm lift coefficient values obtained from simulations were largely varying at angles of attack of $\mathrm{a}=90^{\circ}$ and $0^{\circ}$ for all of the sweepback angles and finger and thumb positions studied. However, for an angle of attack of $\mathrm{a}=45^{\circ}$, and also for all sweepback angles, the lift coefficients attained values much more similar to the hand and forearm drag coefficients, as expected. This similar was particularly evident for sweepback angles of $y=90^{\circ}$ and $270^{\circ}$, at the obtained lift coefficient values were much higher and closer to the drag coefficient values, irrespective of the finger or thumb positions. This finding motivated us to conjecture that, once multiplied by the projected area of the propulsive segment perpendicular to the direction of the considered propulsive force $\left(\mathrm{S}_{\mathrm{L}}\right.$ or $\left.\mathrm{S}_{\mathrm{D}}\right)$, higher lift force values will be produced compared with the drag force at these sweepback angles. Interestingly, the $y=90^{\circ}$ position appears to be the one at which great differences can be observed between swimmers with respect to the relative position of the fingers.

Nevertheless, relevant questions remain to be answered by future research in this domain, such as whether actual swimming conditions allow for effective flow stabilization in each propulsive segment orientation to establish the hydrodynamic settings required for lift creation and maintenance (such as starting vortices and circulation around the hand ${ }^{32}$ ). This matter could not be discussed based on the present results because flow stability was assumed as a condition for the numerical approach.

Previous numerical studies have only analyzed isolated hand models and sweepback angles of $y=0^{\circ} .9,23,24$ Moreover, we attempted to associate different thumb positions with different extents of finger spreading, combining different relative positions of the fingers, in contrast to previous studies that only considered different extents of finger spreading ${ }^{9,16,24}$ or different thumb positions. ${ }^{23}$

The computational fluid dynamics data reported in these previous studies appear to indicate that when the thumb leads the stroke motion (sweepback angle of $\mathrm{y}=0^{\circ}$ ) and, especially during the insweep phases of the stroke, when the lift force might play an important role in overall force production, a hand position with the thumb abducted would be preferable to an adducted thumb position. However, when the drag force plays a major role (angle of attack of $\mathrm{a}=90^{\circ}$ ), positions in which the thumb is adducted appear to benefit swimmer propulsion. ${ }^{23}$ Regarding finger spreading, hand positions with the fingers slightly spread appear to increase the projected area of the hand, thus increasing force production.9,24 Nevertheless, regarding the lift coefficient, the values obtained for the positions with the fingers slightly spread and fingers close together were very similar.

However, when the hand and forearm are both included in simulations, the role of the hand and forearm orientation is much more relevant than the relative position of the fingers. Different sweepback and attack angles produce different hand shapes that affect lift and propulsive drag production in different ways. Indeed, the geometry of the hand circumstantially used by a swimmer, especially the position of the thumb, appears to be dependent on and determined by the predominance of the lift and drag forces in each phase of the propulsive action, aiming to best orient the resultant force and thus the effective propulsive force (the resultant component on the direction of swimming). Thus, thumb abduction and adduction tend to favor propulsive drag or lift under different conditions.

The data presented suggest that the positions with the thumb adducted cause the drag coefficient to increase, as previously indicated by Takagi et $\mathrm{al}^{18}$ for sweepback angles of $\mathrm{y}=0^{\circ}$ and $180^{\circ}$, and by Stewart and Skews, ${ }^{22}$ in contrast to the data of Schleihauf, ${ }^{16}$ who reported higher drag values for the thumb $75 \%$ abducted and $100 \%$ abducted. The lift coefficient presented higher values when the thumb was adducted for sweepback angles of $y=90^{\circ}$ and $270^{\circ}$ and abducted (middle or apart) for sweepback angles of $y=0^{\circ}$ and $180^{\circ}$. These data contrast with those reported by Berger et $\mathrm{al}^{20}$ and Takagi et al, ${ }^{18}$ who indicated higher values for the thumb adducted for a sweepback angle of $y=180^{\circ}$ (although the lift coefficient was higher for a partially abducted position and a sweepback angle of $\mathrm{y}=$ $0^{\circ}$ in the Takagi et $\mathrm{l}^{18}$ study). However, the current CFD data are in accord with those obtained by Marinho et $\mathrm{al}^{23}$ by the hydrodynamic analysis of a swimmer hand simulating a sweepback angle of $y=0^{\circ}$.

Regarding the spreading of the fingers, the main data suggest that the fingers should be grouped or even slightly separated to 
maximize lift and propulsive drag force production for most sweepback and attack angles. This finding is in accord with previously reported experimental ${ }^{16,17,20,21}$ and numerical data..$^{9,24}$

Literature shows no consensus regarding the lift and propulsive drag potential of the hand at different sweepback and attack angles or about the effect of hand shape (especially regarding thumb positions). Different studies have used different methodological procedures (Table 1). Analytical, experimental (using water channels or wind tunnels; different flow velocities), and numerical (using different geometries or boundary conditions) approaches have been adopted in an attempt to understand this interesting and controversial issue, sometimes leading to different results. One very interesting finding of this investigation was that the hand and forearm propulsive segments in swimming allow for similar $C_{L}$ and $C_{D}$ values for different geometries considering fingers and thumb positioning for similar orientations of water flow and attack angles, suggesting that a given swimmer, or different subjects, decides to use different hand geometries at different moments. As a consequence, the standardization of a rule regarding finger and thumb positioning in swimming for a single technique or all of the different available techniques, or in different phases of each technique, does not seem possible, suggesting a point of departure for future detailed and subject-specific training; particular attention should be given by coaches and swimmers to an individual's "water sensitivity".

In summary, finger and thumb positioning in swimming is a determinant of swimming propulsive force production, which is dependent on the direction of the flow over the hand and forearm, changing over the course of the arm's stroke. As a consequence, coaches should be aware that the most appropriate technique must include changes in the relative positions of the fingers and thumbs during the underwater path and that attention should be paid to the training of swimmers' specific sensitivity to the hydrodynamic effects of water flow over the propulsive segments. It can be expected, thus, that changes in the position of the fingers and thumb occur during the arm stroke.

\section{Acknowledgments}

This work was supported by a Grant of the Portuguese Science and Technology Foundation (PTDC/DES/098532/2008, FCOMP-01-0124FEDER-009569).

\section{References}

1. Payton C, Baltzopoulos V, Bartlett R. Contributions of rotations of the trunk and upper extremity to hand velocity during front crawl swimming. J Appl Biomech. 2002;18(3):243-256.

2. Dickinson MH. How animals move: an integrative view. Science. 2000;288:100-106. PubMed doi:10.1126/science.288.5463.100

3. Arellano R, Nicoli-Terrés JM, Redondo JM. Fundamental hydrodynamics of swimming propulsion. In: Vilas-Boas JP, Alves F, Marques A, eds. Proceedings of the $X^{\text {th }}$ International Symposium of Biomechanics and Medicine in Swimming. Portuguese Journal of Sport Sciences; 2006: 15-20.

4. Boulding N, Yim SS, Keshavarz-Moore E, Ayazi Shamlou P, Berry M. Ultra scaledown to predict filtering centrifugation of secreted antibody fragments from fungal broth. Biotechnol Bioeng. 2002;79:381-388. PubMed doi:10.1002/bit.10306

5. Marshall I, Zhao S, Papathanasopoulou P, Hoskins P, Xui Y. MRI and CFD studies of pulsatile flow in healthy and stenosed carotid bifurcation models. J Biomech. 2004;37:679-687. PubMed doi:10.1016/j. jbiomech.2003.09.032
6. Guerra D, Ricciardi L, Laborde JC, Domenech S. Predicting gaseous pollutant dispersion around a workplace. J Occup Environ Hyg. 2007;4:619-633. PubMed doi:10.1080/15459620701484072

7. Dabnichki P, Avital E. Influence of the position of crew members on aerodynamics performance of two-man bobsleigh. J Biomech. 2006;39:2733-2742. PubMed doi:10.1016/j.jbiomech.2005.10.011

8. Lecrivain G, Slaouti A, Payton C, Kennedy I. Using reverse engineering and computational fluid dynamics to investigate a lower arm amputee swimmer's performance. J Biomech. 2008;41:2855-2859. PubMed doi:10.1016/j.jbiomech.2008.06.036

9. Minetti AE, Machtsiras G, Masters JC. The optimum finger spacing in human swimming. J Biomech. 2009;42:2188-2190. PubMed doi:10.1016/j.jbiomech.2009.06.012

10. Marinho DA, Silva AJ, Reis VM, et al. Three-dimensional CFD analysis of the hand and forearm in swimming. $J$ Appl Biomech. 2011;27:74-80. PubMed

11. Lyttle A, Keys M. The application of computational fluid dynamics for technique prescription in underwater kicking. Port J Sport Sci. 2006;6:233-235.

12. von Loebbecke A, Mittal R, Mark R, Hahn J. A computational method for analysis of underwater dolphin kick hydrodynamics in human swimming. Sports Biomech. 2009;8:60-77. PubMed doi:10.1080/14763140802629982

13. Zaidi H, Taiar R, Fohanno S, Polidori G. Analysis of the effect of swimmer's head position on swimming performance using computational fluid dynamics. J Biomech. 2008;41:1350-1358. PubMed doi:10.1016/j.jbiomech.2008.02.005

14. Marinho DA, Reis VM, Alves FB, et al. The hydrodynamic drag during the gliding in swimming. J Appl Biomech. 2009;25:253-257. PubMed

15. Popa CV, Arfaoui A, Fohanno S, Taiar R, Polidori G. Influence of a postural change of the swimmer's head in hydrodynamic performances using 3D CFD. Comput Methods Biomech Biomed Engin. 2014;17(4):344-351. PubMed

16. Schleihauf RE. A hydrodynamic analysis of swimming propulsion. In: Terauds J, Bedingfield EW, eds. Swimming III. Baltimore: University Park Press; 1979:70-109.

17. Remmonds P, Bartlett RM. Effects of finger separation. Swim Tech. 1981;18:28-30.

18. Takagi H, Shimizu Y, Kurashima A, Sanders R. Effect of thumb abduction and adduction on hydrodynamic characteristics of a model of the human hand. In: Blackwell J, Sanders R, eds. Proceedings of Swim Sessions of the XIX In International Symposium on Biomechanics in Sports. San Francisco: University of San Francisco; 2001: 122-126.

19. Berger MA, de Groot G, Hollander AP. Hydrodynamic drag and lift forces on human hand arm models. J Biomech. 1995;28:125-133. PubMed doi:10.1016/0021-9290(94)00053-7

20. Berger MA, de Groot G, Hollander AP. Influence of hand shape on force generation during swimming. In: Eriksson BO, Gullstrand L, eds. Proceedings of the XII FINA World Congress on Sports Medicine. Goteborg: Chalmers Reproservice; 1997: 389-396.

21. Sidelnik NO, Young BW. Optimising the freestyle swimming stroke: the effect of finger spread. Sports Eng. 2006;9:129-135. doi:10.1007/ BF02844114

22. Stewart A, Skews BW. Forces on the swimming hand. $R$ J. 2002;18:70 76.

23. Marinho DA, Rouboa AI, Alves FB, et al. Hydrodynamic analysis of different thumb positions in swimming. J Sports Sci Med. 2009;8:5866. PubMed

24. Marinho DA, Barbosa TM, Reis VM, et al. Swimming propulsion forces are enhanced by a small finger spread. J Appl Biomech. 2010;26:87-92. PubMed

25. Lauder M, Dabnichki P, Bartlett R. Improved accuracy and reliability of sweepback angle, pitch angle and hand velocity calculations in 
swimming. J Biomech. 2001;34:31-39. PubMed doi:10.1016/S00219290(00)00166-4

26. Counsilman JE. The application of Bernoulliís Principle to Human Propulsion in Water. In: Lewillie L, Clarys J, eds. First International Symposium on Biomechanics of Swimming. Universite Libre de Bruxelles, Brussels, Belgium; 1971: 59-71.

27. Wood TC. A fluid dynamic analysis of the propulsive potential of the hand and forearm in swimming. Master of Science Thesis, Dalhouise University Press, Halifax. 1977.

28. Maglischo EW. Swimming Faster. California: Mayfield Publishing Company; 1982
29. Rushall BS, Holt LE, Sprigins EJ, Cappaert J. A re-evaluation of forces in swimming. J Swim Res. 1994;10:6-30.

30. Maglischo EW. Biomechanics. Newsletter of the world swimming coaches Association. 1999; 99: 1-2.

31. Maglischo EW. Swimming fastest. Champaign, Illinois: Human Kinetics; 2003.

32. Douglas PF, Gasiorek JM, Swaffienl JA. Fluid mechanics. London: Pitman; 1979. 